\title{
Commentary article
}

\section{Nonprescription antibiotic sales in China and the implications}

\author{
Kalungia $A^{1}$, Godman $B^{2,3,4,5^{\star}}$ \\ 1Department of Pharmacy, University of Zambia, Lusaka, Zambia. Email: ckalungia@unza.zm \\ ${ }^{2}$ Strathclyde Institute of Pharmacy and Biomedical Sciences, University of Strathclyde, Glasgow G4 \\ ORE, United Kingdom. Email: brian.godman@strath.ac.uk \\ ${ }^{3}$ Division of Clinical Pharmacology, Karolinska Institute, Karolinska University Hospital Huddinge, SE- \\ 141 86, Stockholm, Sweden. Email: Brian.Godman@ki.se \\ ${ }^{4}$ Department of Pharmacy, Faculty of Health Sciences, Sefako Makgatho Health Sciences University, \\ Pretoria, South Africa \\ ${ }^{5}$ Health Economics Centre, University of Liverpool Management School, Liverpool, UK. Email: \\ Brian.Godman@liverpool.ac.uk
}

*Author for correspondence: Strathclyde Institute of Pharmacy and Biomedical Sciences, University of Strathclyde, Glasgow G4 ORE, United Kingdom. Email: Brian.godman@strath.ac.uk. Telephone: 0141548 3825. Fax: 01415522562 and Division of Clinical Pharmacology, Karolinska Institute, Karolinska University Hospital Huddinge, SE-141 86, Stockholm, Sweden. Email: Brian.Godman@ki.se. Telephone + 46858581068 . Fax + 46859581070

(Accepted for publication Lancet Infectious Diseases - Please keep Confidential)

The paper by Jie Chang and colleagues published in The Lancet Infectious Diseases is very timely (1). This reflects a critical need for concerted efforts to reduce irrational use of antibiotics to lower rising antimicrobial resistance (AMR) rates (2-4), with their overuse resulting in antibiotics becoming the most commonly used medicines globally $(3,5)$. AMR increases morbidity, mortality, and costs as health systems run out of antimicrobial options to treat common infectious diseases $(1,2,6,7)$. Lower and middle-income countries (LMICs) including China are a particular concern given their increasing overuse of antibiotics (8) coupled with a greater impact of AMR due to issues including poor sanitation, malnutrition and dense populations (8).

A critical issue among LMICs is inappropriate non-prescription antibiotic sales especially for selflimiting diseases such as diarrhoea and upper respiratory tract infections (URTIs) of viral aetiology (1, $3-5,7,8)$. Concerns are exacerbated by often shorter courses and inappropriate antibiotics being dispensed (3). Overall, rates of non-prescription sales of antibiotics in LMICs are up to $93 \%$ of dispensed antibiotics, with up to $100 \%$ of pharmacists dispensing antibiotics without a prescription in some LMICs despite legislation against such practises $(1,4,5,8,9)$. Ours and others' experience is that non-prescription antibiotic sales is driven by multiple reasons including pressure from patients and financial reasons, i.e. profit-motivated community pharmacists fearing losing clients seeking antibiotics to competitors and high rates of self-medication where patients cannot afford physician fees and opt straight for out-of-pocket purchases. Ease of access to antibiotics and weak enforcement of regulations also enhance non-prescription sales. Easier access of community pharmacies as opposed to physicians in primary healthcare centres with often medicine shortages, coupled with cultural issues and poor levels of education $(1,5,8)$, also influences patients' behaviour.

Consequently, community pharmacists are a critical component to improving the management of patients with URTIs and paediatric diarrhoea especially as they are often the first healthcare professional patients consult $(1,10)$. However, there can be concerns with their level of knowledge regarding antibiotics, antimicrobial stewardship (AMS), and $\operatorname{AMR}(1,11,12)$. Initiatives are needed to address this.

The comprehensive approach by Chang et al using simulated clients (SCs) provides further insight into current rates of non-prescription sales of antibiotics in China where the authorities are seeking to change behaviour (1). Using simulated clients helps gain an accurate insight into not only pharmacists' but also other health professionals' behaviour towards antibiotics $(1,8,10)$. Whilst there are still concerns with the high rate of non-prescription sales of antibiotics among community pharmacists across China at $48.5 \%$ and $70.1 \%$ for paediatric diarrhoea and URTIs, respectively, and only a limited number asked the simulated clients about taking other medicines (6.6\% to $9.7 \%)$ or 
their allergies and issuing advice on medicine taking (16.1\% to $29.2 \%)$, there were encouraging signs (1). These include the fact that sales were significantly less common in urban areas, having a pharmacist on duty and being part of a pharmacy chain (1). In addition, multiple initiatives in Shaanxi including stricter regulations for dispensing antibiotics, a qualified pharmacist's presence to dispense antibiotics, increased frequency of unannounced pharmacy inspections, punishments for misuse, and improving pharmacists' education, showed decreasing antibiotic sales between 2011 and 2017 (1).

As a result of the findings of Chang et al and our experience, we believe several strategies can be implemented to reduce non-prescription antibiotic sales. These include improving pharmacists' knowledge and practice of AMS starting in pharmacy school coupled with the use of the Internet and educational workshops post qualification (1), making pharmacists more aware of the WHO AWaRe antibiotic list especially which antibiotics not to dispense, as well as developing and implementing national guidelines for pharmacists $(1,4,13)$. Other activities including reinforcing legislation where pertinent, instigating IT surveillance systems to track antibiotics through the supply chain, monitoring pharmacy activities using mobile technologies and increased patient education on the dangers of selfmedication with antibiotics. Multifaceted programmes are typically needed to change behaviour. Notwithstanding that community health campaigns are more challenging in LMICs (14), by championing pharmacists as antibiotic guardians they can take the lead to improving antibiotic use in the community and reduce AMR rates.

In conclusion, the findings by Chang and colleagues do provide guidance in this crucial area, and we will be monitoring developments in the future to reduce AMR rates.

\section{Author contributions}

Both authors contributed fully to the production of this commentary paper. Both authors approved the final submission.

\section{Conflicts of interest}

The authors have no conflicts of interest to declare

\section{Funding}

There has been no funding for this commentary paper

\section{References}

1. Chang J, Xu S; Zhu S, Li Z, Yu J, Zhang Y et al. Using Simulated Clients to Assess Nonprescription Antibiotic Dispensing at Community Pharmacies in China: A Mixed Cross-sectional and Longitudinal Study. The Lancet Infectious Diseases. 2019. In Press

2. O'Neill J. Antimicrobial Resistance: Tackling a crisis for the health and wealth of nations. The Review on Antimicrobial Resistance. 2014. Available at URL: https://amrreview.org/sites/default/files/AMR\%20Review\%20Paper\%20\%20Tackling $\% 20$ a $\% 20$ crisis $\% 20$ for $\% 20$ the $\% 20$ health $\% 20$ and $\% 20$ wealth $\% 20$ of $\% 20$ nations 1.pdf. 3. Auta A, Hadi MA, Oga E, Adewuyi EO, Abdu-Aguye SN, Adeloye D, et al. Global access to antibiotics without prescription in community pharmacies: A systematic review and meta-analysis. The Journal of infection. 2019;78(1):8-18.

4. Torres NF, Chibi B, Middleton LE, Solomon VP, Mashamba-Thompson TP. Evidence of factors influencing self-medication with antibiotics in low and middle-income countries: a systematic scoping review. Public health. 2019;168:92-101.

5. Nepal G, Bhatta S. Self-medication with Antibiotics in WHO Southeast Asian Region: A Systematic Review. Cureus. 2018;10(4):e2428.

6. Founou RC, Founou LL, Essack SY. Clinical and economic impact of antibiotic resistance in developing countries: A systematic review and meta-analysis. PloS one. 2017;12(12):e0189621. 7. Rather IA, Kim BC, Bajpai VK, Park YH. Self-medication and antibiotic resistance: Crisis, current challenges, and prevention. Saudi journal of biological sciences. 2017;24(4):808-12.

8. Sakeena MHF, Bennett AA, McLachlan AJ. Non-prescription sales of antimicrobial agents at community pharmacies in developing countries: a systematic review. International journal of antimicrobial agents. 2018;52(6):771-82.

9. Kalungia AC, Burger J, Godman B, Costa JO, Simuwelu C. Non-prescription sale and dispensing of antibiotics in community pharmacies in Zambia. Expert review of anti-infective therapy. 2016;14(12):1215-23. 
10. Markovic-Pekovic V, Grubisa N, Burger J, Bojanic L, Godman B. Initiatives to Reduce Nonprescription Sales and Dispensing of Antibiotics: Findings and Implications. J Res Pharm Pract. 2017;6(2):120-5.

11. Hadi MA, Karami NA, Al-Muwalid AS, Al-Otabi A, Al-Subahi E, Bamomen A, et al. Community pharmacists' knowledge, attitude, and practices towards dispensing antibiotics without prescription (DAwP): a cross-sectional survey in Makkah Province, Saudi Arabia. International journal of infectious diseases. 2016;47:95-100.

12. Hoxha I, Malaj A, Kraja B, Bino S, Oluka M, Markovic-Pekovic V, et al. Are pharmacists' good knowledge and awareness on antibiotics taken for granted? The situation in Albania and future implications across countries. Journal of global antimicrobial resistance. 2018;13:240-5.

13. Sharland M, Pulcini C, Harbarth S, Zeng M, Gandra S, Mathur S, et al. Classifying antibiotics in the WHO Essential Medicines List for optimal use-be AWaRe. The Lancet Infectious diseases. 2018;18(1):18-20.

14. Dar OA, Hasan R, Schlundt J, Harbarth S, Caleo G, Dar FK, et al. Exploring the evidence base for national and regional policy interventions to combat resistance. Lancet.

2016;387(10015):285-95. 